\title{
Recursos Educacionais Multiplataformas Abertos na Rede
}

\author{
Delano M. Beder ${ }^{1,2}$ Joice L. Otsuka ${ }^{1,2}$ Denis Cappelini ${ }^{1,2}$ \\ Matheus V. Fernandes ${ }^{1,2}$ Rener B. da Silva ${ }^{1,2}$ Alex R. Guido ${ }^{2}$ \\ ${ }^{1}$ Laboratório de Objetos de Aprendizagem (LOA) \\ ${ }^{2}$ Departamento de Computação (DC) \\ Universidade Federal de São Carlos (UFSCar) \\ Contato:delano@dc.ufscar.br
}

\begin{abstract}
Resumo. O projeto Recursos Educacionais Multiplataformas Abertos na Rede (REMAR) tem como objetivo facilitar e ampliar a construção e o reuso de recursos educacionais abertos (REA), por meio de uma plataforma web, que ofereça ferramentas que facilitem a construção e a customização de REA, seguindo diretrizes que favoreçam o reuso, a disponibilização desses recursos em diferentes plataformas, bem como a integração com ambientes virtuais de aprendizagem.
\end{abstract}

\begin{abstract}
The Open Multiplataform Educational Resources on the Network (REMAR) project aims to make easier and expand the construction and reuse of open educational resources (OER), through a web platform that offers tools to facilitate the construction and customization of REA, following guidelines that promote the reuse, the publication of these REA on different platforms, as well as the integration with virtual learning environments.
\end{abstract}

\section{Introdução}

O acesso aberto a recursos educacionais é um requisito essencial para a educação democrática, de qualidade, sustentável e aberta, em qualquer modalidade (presencial, a distância, híbrida) e em todos os níveis de formação. Além do acesso, o reuso de recursos educacionais deve ser promovido, considerando que o desenvolvimento desses recursos, em geral, é um processo bastante dispendioso e requer um trabalho conjunto entre especialistas do conteúdo e uma equipe multidisciplinar, sobretudo quando consideramos os recursos educacionais interativos e que integram diferentes mídias e tecnologias.

Neste sentido, o desenvolvimento e o compartilhamento dos denominados Recursos Educacionais Abertos (REA) - que são recursos de acesso aberto que podem ser revisados, customizados, reutilizados e redistribuídos - deve ser amplamente promovido e apoiado [Amiel et al. 2011]. No entanto, o efetivo reuso de um REA envolve questões que vão além de sua disponibilização sob licenças menos restritivas como o Creative Commons, considerando que muitas vezes, apesar de abertos, esses recursos são disponibilizados em formatos que não favorecem a sua adaptação (formatos não editáveis) ou dependentes de ferramentas proprietárias. Além disso, o reuso desses recursos deve ser possível em diferentes níveis, para diferentes perfis de usuário: (i) a desenvolvedores de REA, que devem ter acesso ao código fonte e aos componentes dos REA, podendo adaptá-los a novos contextos e requisitos de uso; (ii) a educadores de diferentes áreas, que devem ter acesso a um conjunto de ferramentas que facilitem a adaptação desses recursos para as suas necessidades, bem como a distribuição aos estudantes, por meio de diferentes plataformas de hardware e software. 
CBIE-LACLO 2015

Anais dos Workshops do IV Congresso Brasileiro de Informática na Educação (CBIE 2015)

Nesse contexto, esse artigo apresenta o projeto Recursos Educacionais Multiplataforma Abertos na Rede (REMAR) que tem como objetivo facilitar e ampliar a construção e o reuso de recursos educacionais abertos (REA), por meio de uma plataforma web, que ofereça ferramentas que facilitem a construção e a customização de REA, seguindo diretrizes que favoreçam o reuso, a disponibilização desses recursos em diferentes plataformas, bem como a integração com ambientes virtuais de aprendizagem.

\section{Plataforma: Visão Geral}

Essa seção apresenta uma visão geral da plataforma proposta que visa facilitar e promover a criação, adaptação e reuso de REA. A solução proposta tem como diferencial ser totalmente aberta, multiplataforma, além de preconizar o reuso e a acessibilidade.

Espera-se que a plataforma proposta possa guiar a construção de REA que possam ser adaptados diretamente pelos professores, de acordo com as suas necessidades e sem a necessidade de conhecimentos específicos de programação. Também espera-se possibilitar a geração de REA em diferentes plataformas, bem como a integração destes ao ambiente virtual de aprendizagem Moodle. Figura 1 ilustra uma visão geral da plataforma proposta. Conforme pode-se observar, a plataforma tem como público-alvo três perfis de usuário: (i) desenvolvedores de novos REA; (ii) professores que queiram adaptar REA criados utilizando a plataforma REMAR; (iii) estudantes que acessam os REA criados em diferentes plataformas.

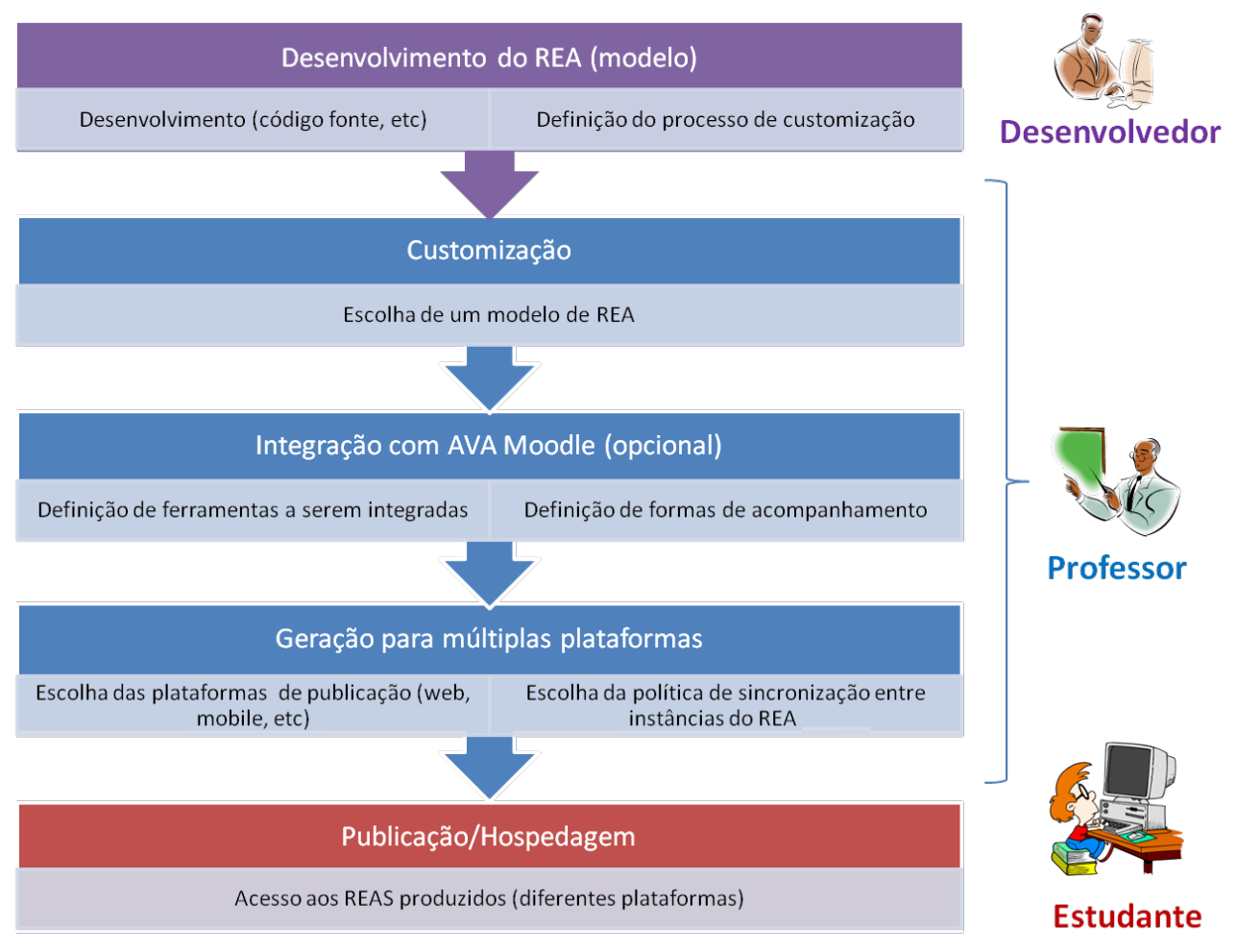

Figura 1. Plataforma REMAR: Visão Geral

Dessa forma, planeja-se obter os seguintes resultados: um conjunto de diretrizes para o desenvolvimento de REA reutilizáveis, adaptáveis, multiplataforma e acessíveis e 


\section{CBIE-LACLO 2015}

Anais dos Workshops do IV Congresso Brasileiro de Informática na Educação (CBIE 2015)

um protótipo da plataforma que disponibilizará as seguintes funcionalidades: (i) autoria dos REA com pontos de customização bem definidos; (ii) customização dos REA, possibilitando a adaptação desses recursos diretamente pelo professor; (iii) geração dos REA para diferentes plataformas (desktop, móvel e web); (iv) sincronização entre as instâncias dos REA publicados em diferentes plataformas; e (v) módulos de integração dos REA com o ambiente virtual de aprendizagem Moodle.

Um esboço da arquitetura da plataforma proposta é apresentado na Figura 2. Quanto à tecnologia para o desenvolvimento da plataforma, foi adotado o framework de desenvolvimento de aplicações web Grails ${ }^{1}$, que é baseado no padrão arquitetural MVC e objetiva a alta produtividade no desenvolvimento de aplicações web.

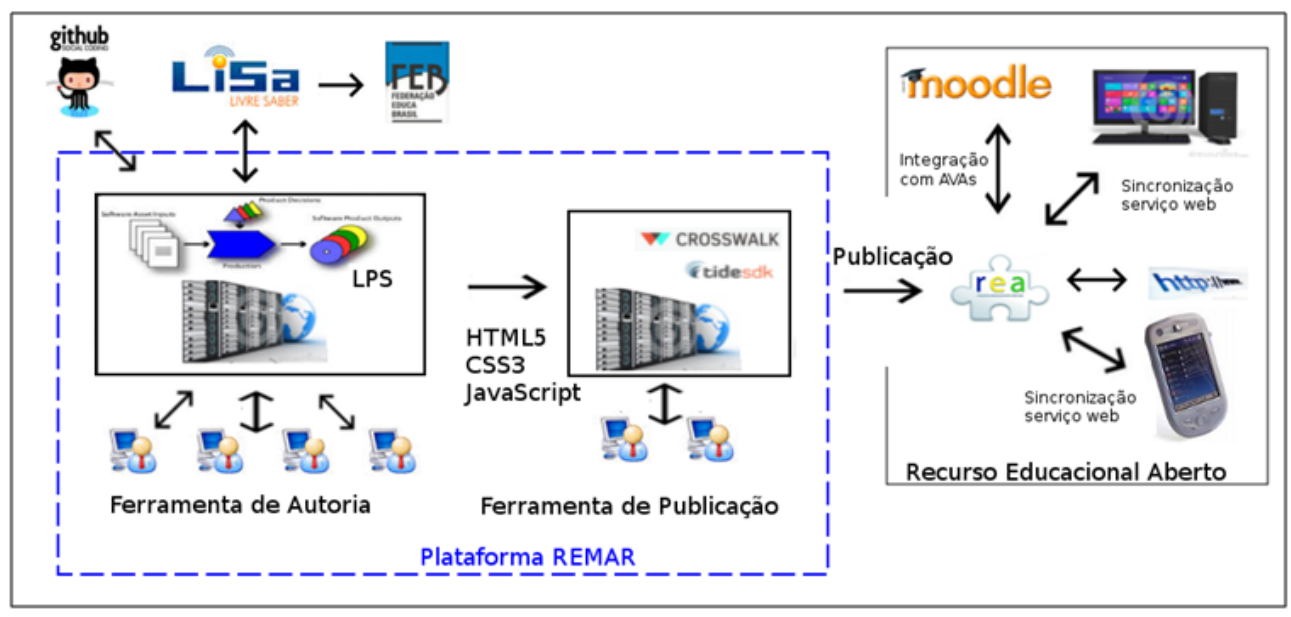

Figura 2. Plataforma REMAR: Esboço da Arquitetura

No contexto da presente proposta, os REA deverão ser projetados seguindo as diretrizes da abordagem de Linha de Produtos de Software (LPS) [Schmid \& de Almeida 2013]. Para tal, a plataforma proposta, por meio da ferramenta de autoria, disponibilizará um conjunto de funcionalidades responsáveis pela definição de Linhas de Produtos de Software, bem como a posterior instanciação de REA similares por meio da escolha de um conjunto comum e gerenciado de funcionalidades (features) e variantes das LPS definidas. Em adição, a ferramenta de autoria proposta permitirá que novas features e/ou variantes das LPS sejam criadas e posteriormente armazenadas em repositórios de acesso livre.

Em adição, a plataforma incorpora, na ferramenta de publicação proposta, as funcionalidades providas pelas ferramentas Crosswalk ${ }^{2}$ e TideSDK $^{3}$ visando a produção e publicação de instâncias dos REA para plataformas web, móvel, desktop. A utilização dessas ferramentas possibilitará a criação de aplicativos (móveis e desktop) nativos destas plataformas a partir de aplicações web desenvolvidas em HTML5.

\section{Resultados iniciais}

O desenvolvimento do processo de personalização e publicação dos primeiros REA possibilitou a validação do uso da plataforma proposta no desenvolvimento de recursos educa-

\footnotetext{
${ }^{1}$ https://grails.org/

${ }^{2}$ https://crosswalk-project.org/

${ }^{3}$ http://www.tidesdk.org/
} 
CBIE-LACLO 2015

Anais dos Workshops do IV Congresso Brasileiro de Informática na Educação (CBIE 2015)

cionais abertos. Figura 3 ilustra a visão de um usuário com perfil de professor. Conforme pode-se observar, essa página apresenta a lista dos recursos educacionais possíveis de serem personalizados pela plataforma proposta.

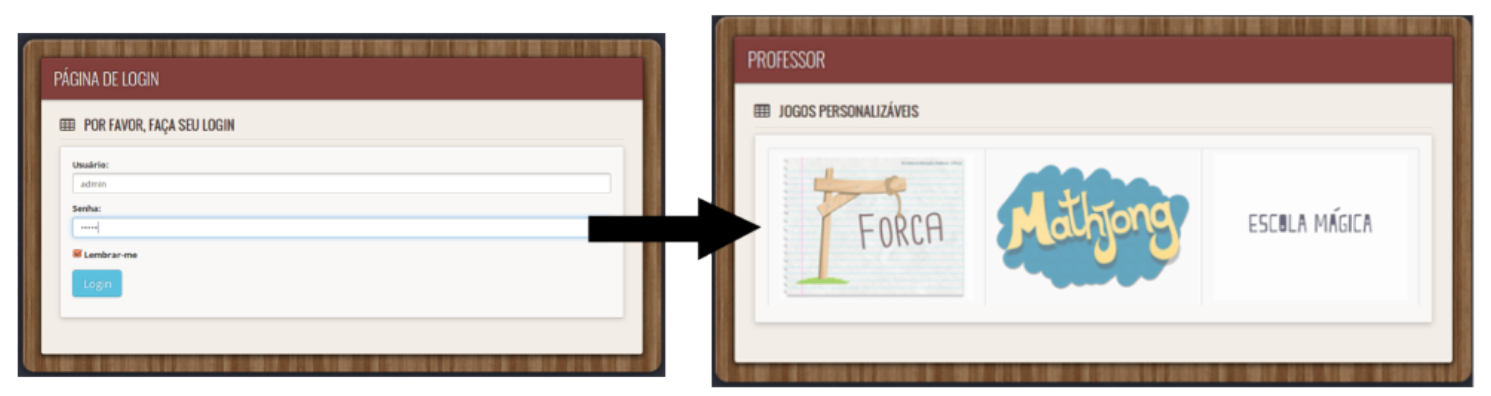

Figura 3. Perfil professor: lista de recursos educacionais

Por questões de brevidade, essa seção descreve apenas uma das provas de conceito realizadas: a personalização e publicação do jogo MathJong. O MathJong é baseado no MahJong e tem como objetivo trabalhar operações e precedência de operadores matemáticos. O jogador tem como objetivo encontrar pares, conectados por máximo 3 linhas, de expressões matemáticas equivalentes (mesmo valor) ${ }^{4}$.

Conforme discutido, no contexto da plataforma REMAR, os REA deverão ser projetados seguindo as diretrizes da abordagem de Linha de Produtos de Software (LPS). Nesse contexto, por exemplo, a mecânica do MathJong, poderia ser considerada como uma funcionalidade comum (feature) enquanto as expressões matemáticas utilizadas poderiam ser consideradas como a variante dessa LPS.
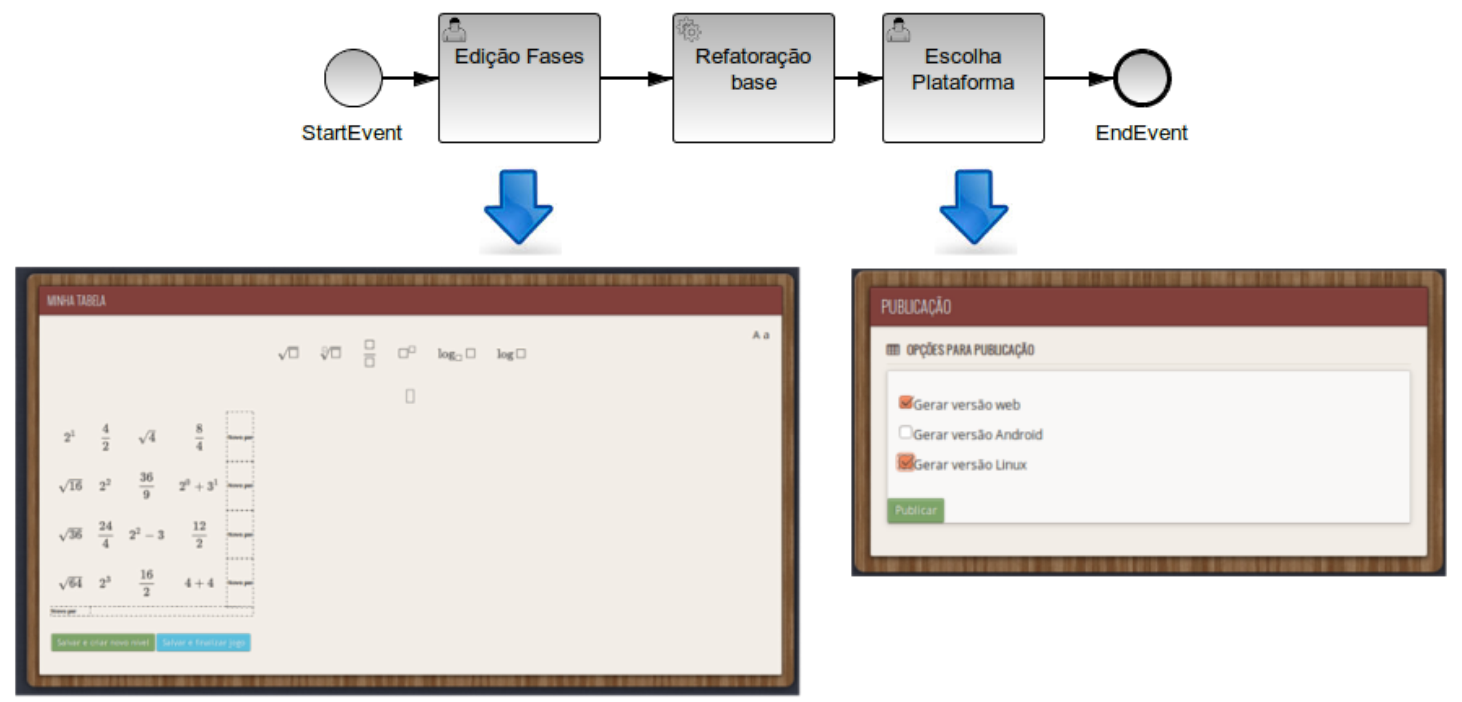

Figura 4. Modelo BPMN de autoria e publicação do REA MathJong

Figura 4 ilustra o processo de negócio, utilizando a notação BPMN [Ko 2009], relacionado às modificações necessárias nas partes variantes dessa LPS bem como a publicação das diferentes instâncias desse REA em múltiplas plataformas. A plataforma

\footnotetext{
${ }^{4}$ Ou seja, segundo a dinâmica do jogo Mahjong (http://www.ojogos.com.br/jogo/Mahjong-Link.html)
} 
CBIE-LACLO 2015

Anais dos Workshops do IV Congresso Brasileiro de Informática na Educação (CBIE 2015)

REMAR utiliza o $B P M S$ Camunda ${ }^{5}$ para dar apoio à automatização dos processos $B P M N$. Dessa forma, essa figura ilustra também as páginas web que são abertas, pelo BPMS Camunda, durante a personalização do REA: (i) um editor de expressões matemáticas em que o usuário com papel de professor pode definir as expressões que serão utilizadas no REA personalizado; (ii) uma página web em que são definidos em quais plataformas o REA será publicado.

Por fim, Figura 5 ilustra a visão de um usuário com perfil de estudante. Conforme pode-se observar, essa página apresenta o REA MathJong personalizado com as expressões definidas anteriormente pelo usuário com perfil de professor.

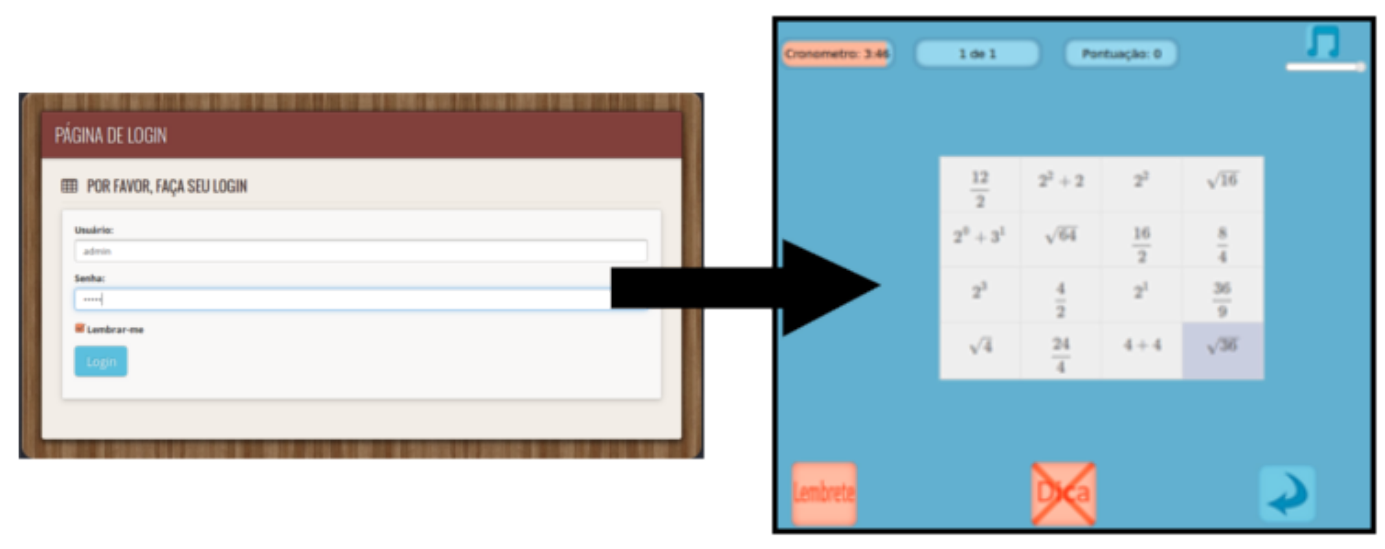

Figura 5. Perfil Estudante: instância do REA MathJong personalizado

\section{Considerações finais}

Neste artigo foram discutidas as bases, os objetivos e resultados iniciais da iniciativa do projeto REMAR para o desenvolvimento de recursos educacionais abertos. A plataforma proposta tem como potencial prover um serviço que facilitará a geração e adaptação de REA em múltiplas plataformas, por meio de ferramentas que poderão ser utilizadas por desenvolvedores (criação de novos REA) e não desenvolvedores (adaptação, geração de instâncias em múltiplas plataformas). Dessa forma, espera-se contribuir para a produção e uso de REA em todas as modalidades de ensino (presencial, a distância, híbrida), em todos os níveis de formação.

\section{Agradecimentos}

Os autores agradecem o apoio e suporte concedido pela Rede Nacional de Pesquisa (RNP) e pela Coordenação de Aperfeiçoamento de Pessoal de Nível Superior (CAPES).

\section{Referências}

Amiel, T., Orey, M., \& West, R. (2011). Recursos educacionais abertos: Modelos para localização e adaptação. Educação Temática Digital.

Ko, R. K. L. (2009). A Computer Scientist's Introductory Guide to Business Process Management (BPM). Crossroads, 15(4):4:11-4:18.

Schmid, K. \& de Almeida, E. (2013). Product Line Engineering. IEEE Software, 30(4).

\footnotetext{
${ }^{5}$ https://camunda.com/
} 